\title{
Development of New Attachment System with Soft Lining Material for Implant-retained Complete Denture
}

\author{
Takashi Koike, Takayuki Ueda, Syuutarou Noda, Koichiro Ogami, Pravinkumar G Patil, Kaoru Sakurai
}

\begin{abstract}
Purpose: The retention of complete dentures is frequently difficult in edentulous patients with a severely resorbed residual alveolar ridge. In such cases, implant overdentures having a stronger retention force are useful. Although there are various types of attachment systems for implant overdentures, they are frequently complicated. We are, therefore, developing a simple attachment system focusing on a soft lining material for the female connector. The purpose of this study was to evaluate the retention force of implant overdentures applying a soft lining material for the attachment.
\end{abstract}

Materials and methods: The models in which seven male and female connector pairs were used. As the control, a rubber O-ring attachment system, in which a rubber O-ring female was paired with a metal ball anchor (male), having a diameter of 1.7 or $2.2 \mathrm{~mm}$. In the attachment system with a soft lining material, a female component was made up of soft lining material paired with a male having a diameter of $1.7,2.2,2.5,2.7$ or $3.0 \mathrm{~mm}$. In measuring the retention force, the maximum force required to dislodge the male from the female was recorded by a digital force gauge.

Results: With the rubber O-ring, the retention force was $2.9 \pm$ $0.2 \mathrm{~N}$ for the $1.7 \mathrm{~mm}$ male and $3.3 \pm 0.3 \mathrm{~N}$ for the $2.2 \mathrm{~mm}$ male; these values were higher than the respective retention forces when using a soft lining material as the female and a male of the same diameter. However, with the female comprising soft lining material, the retention force exceeded $4 \mathrm{~N}$ for the 2.5, 2.7 and $3.0 \mathrm{~mm}$ males and was higher than the retention forces for the rubber O-ring.

Conclusion: The proposed attachment system with soft lining material for implant overdentures provides a clinically sufficient retention force when the diameter of the male exceeds $2.5 \mathrm{~mm}$.

Keywords: Implant-retained complete denture, Implant supported overdentures, Soft lining material, Attachment system, Retention.

How to cite this article: Koike T, Ueda T, Noda S, Ogami K, Patil PG, Sakurai K. Development of New Attachment System with Soft Lining Material for Implant-retained Complete Denture. Int J Prosthodont Restor Dent 2013;3(1):21-24.

Source of support: Nil

Conflict of interest: None

\section{INTRODUCTION}

For completely edentulous patients, removable implant overdentures (IODs) have many advantages over fixed restorations in regards to lesser number of implants required and easy to keep the restorations clean by the patients. The McGill consensus on IOD was published, ${ }^{1}$ which proposed that mandibular two-implant overdentures should be considered as the standard of care for edentulous patients.
Thereafter, IODs have been used worldwide ${ }^{2}$ and will be further employed in the future.

However, there is no attachment system for IOD that can be considered the gold standard. Although many excellent attachment systems have been put into practice, each system has its own problems. For example, wear of ball attachments with a metal female connector ${ }^{3}$ decreases the retention. When the placement angle of fixtures is nonparallel, the male connector is abraded. Bar attachments require a wide space to insert the bar inside overdentures. Magnetic attachments require the uniform contact of all the magnets and keepers. Because of these problems, in some cases, a stricter fixture placement angle is required in comparison with the case for implant-supported fixed dental prostheses. Furthermore, regarding the design of dentures, the incorporation of attachments into dentures is made difficult by the tooth arrangement restricting the space required. Therefore, improvements of the junction area of fixtures and dentures are needed. Attachments require a wide range of insertion and detachment directions, low abrasion of the male connector inserted in fixtures and for the abrasion to be easily repaired, the size of the attachment appliance to be as small as possible, narrow incorporation space inside the dentures and a simple manipulation technique.

To realize these improvements, focusing on soft lining materials, we are developing a retention system for overdentures, using a soft lining material as the female connector and a ball anchor as the male connector. The physical properties of soft lining materials decrease the burden on fixtures due to occlusal forces. ${ }^{4,5}$ This study, therefore, evaluates the retention force to verify the usefulness of the present system in which a soft lining material is used to connect implants and overdentures.

\section{MATERIALS AND METHODS}

Using models in which seven male connector and female connector pairs were combined, the retention force was measured. As the control, a rubber O-ring attachment system (OP Anchor attachment, Hakuho, Japan) was used. A metal ball anchor with diameter of $1.7 \mathrm{~mm}$ (R-1.7) or $2.2 \mathrm{~mm}$ (R-2.2) was paired with the female connector of the rubber O-ring. In the attachment system with soft lining material, the soft lining female connector was paired with a metal ball anchor (male connector) having a diameter of $1.7 \mathrm{~mm}$ (S-1.7) or $2.2 \mathrm{~mm}$ (S-2.2), or a handmade male connector 
having a diameter of $2.5 \mathrm{~mm}(\mathrm{~S}-2.5), 2.7 \mathrm{~mm}(\mathrm{~S}-2.7)$ or $3.0 \mathrm{~mm}$ (S-3.0) (Figs 1A to $\mathrm{G}$ ).

On plaster models imitating the residual alveolar ridge, experimental bases imitating overdentures were produced (Figs 2A to C). Using heat-polymerized acrylic resin (GC Acron No.3, GC Corporation, Japan), the experimental base was produced according to the method described by the manufacturers. One attachment male connector was fixed in the plaster model. The experimental base was fitted on the upper surface of the plaster model and a space $(7 \mathrm{~mm}$ in diameter and $5 \mathrm{~mm}$ deep) to incorporate the male connector was established. After applying a separating medium to the upper surface of the plaster model, a soft lining material (Sofreliner Tough Medium; Tokuyama Dental, Japan) was set and pressure $(5 \mathrm{~N})$ was applied for 7 minutes (Fig. 3A). The rubber O-ring was fixed to the space in the experimental base using autopolymerizing acrylic resin (Unifast III; GC, Japan) (Fig. 3B).

To measure the retention force, after dripping artificial saliva $^{6}(60 \%$ glycerin solution) on the plaster model, the experimental base was inserted, and pressure $(5 \mathrm{~N})$ was applied for 10 seconds. Thereafter, the experimental base was pulled in the vertical direction at a cross-head speed of $25 \mathrm{~mm} / \mathrm{mins}$ (Fig. 4). The maximum force required to detach the experimental base from the plaster model was measured 10 times, and the mean value was taken as the retention force. Data were analyzed employing the Bonferroni test for multiple comparisons $(\alpha=0.05)$.

\section{RESULTS}

The retention force of the rubber O-ring was $2.9 \pm 0.2 \mathrm{~N}$ for $\mathrm{R}-1.7$ and $3.3 \pm 0.3 \mathrm{~N}$ for $\mathrm{R}-2.2$; these values significantly differ from values for $\mathrm{S}-1.7(0.5 \pm 0.2)$ and $\mathrm{S}-2.2(0.7 \pm 0.1)$ with soft lining material female connectors, respectively. In the case of soft lining material, the retention force exceeded $4 \mathrm{~N}$ for S-2.5, S-2.7 and S-3.0; these values significantly differ from values for $\mathrm{S}-1.7$ and $\mathrm{S}-2.2$. Furthermore, retention forces for S-2.5, S-2.7 and S-3.0 significantly differ from values for R-1.7 and R-2.2 (Fig. 5).

\section{DISCUSSION}

The O-ring attachment system has long been used for toothsupported overdentures, ${ }^{7,8}$ providing clinically favorable results. Furthermore, it is currently used for the system of IOD, and there have been reports ${ }^{9-11}$ presenting favorable results. Therefore, if the present attachment system, which is being developed in this study, can achieve a retention force equal to or higher than that of the O-ring attachment system, it is considered that the present system will become clinically applicable. To achieve a sufficient retention force using a soft lining material, ball anchors with diameters of 2.5, 2.7 and $3.0 \mathrm{~mm}$ were produced and experimentally investigated in the present paper. Although it was possible to produce larger male connectors, the male connector size is clinically restricted. This is because implant placement techniques become complicated when the diameter of attachments exceeds that of implants in the one-piece implant system, such as in the case of mini-implants whose clinical cases ${ }^{12}$ and usefulness in preventing alveolar ridge resorption ${ }^{13}$ have been reported in recent years. The crosshead speed in this study was set at $25 \mathrm{~mm} / \mathrm{min}$. This was because measurements varied little at this traction speed in preliminary studies, and this traction speed was found to be stable in previous studies. ${ }^{14}$

Because the results obtained in this study of the retention force for R-1.7 and R-2.2 were higher than those obtained for S-1.7 and S-2.2, respectively; it is noted that a sufficient retention force cannot be achieved by only replacing the O-ring of the O-ring attachment system with the soft lining material. Retention forces of S-2.5, S-2.7 and S-3.0 were higher than those of R-1.7 and R-2.2. It was thus confirmed that, although the retention force of the soft lining material is apt to be low owing to the material's low hardness, a retention force higher than that of the rubber O-ring attachment system can be achieved by increasing the diameter of the female connector. Regarding the relationship between the morphology of the male connector and retention force, it is considered that the increase in the diameter is more important than that of the height in improving the retention
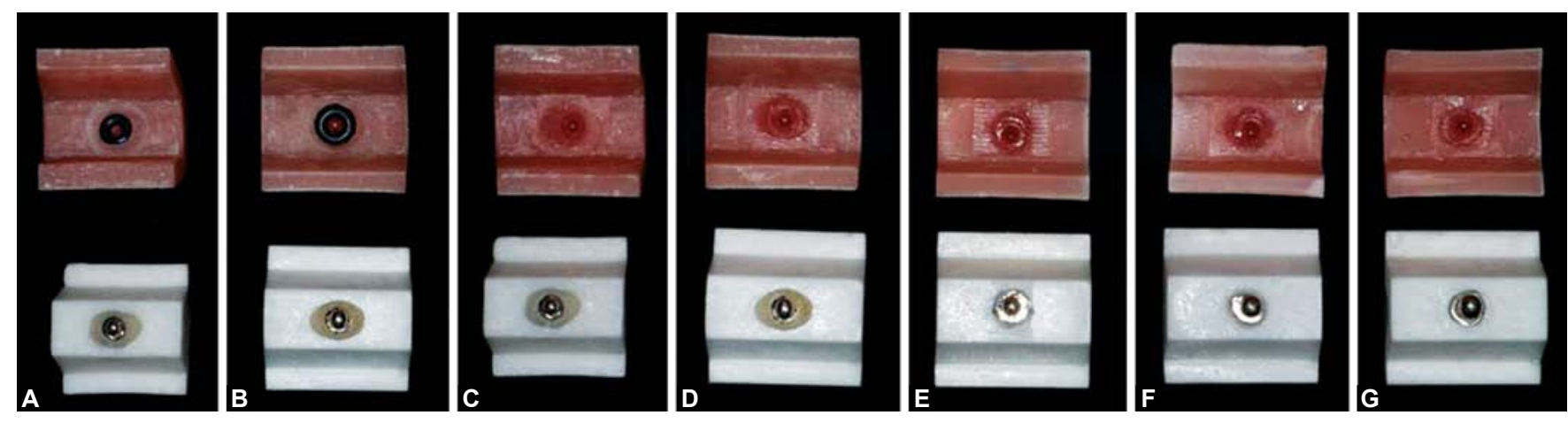

Figs 1A to G: List of male and female connector pairs used in this experiment 


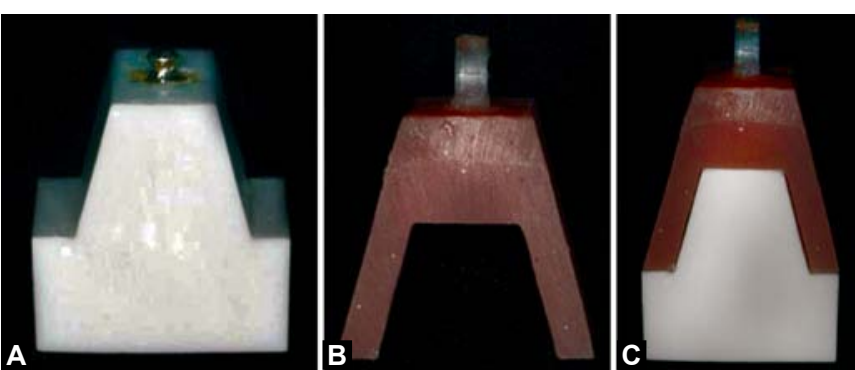

Figs 2A to C: Measuring device: $(A)$ male connector set on the upper surface of the plaster model imitating the residual alveolar ridge; (B) experimental base and $(C)$ experimental base combined with the plaster model
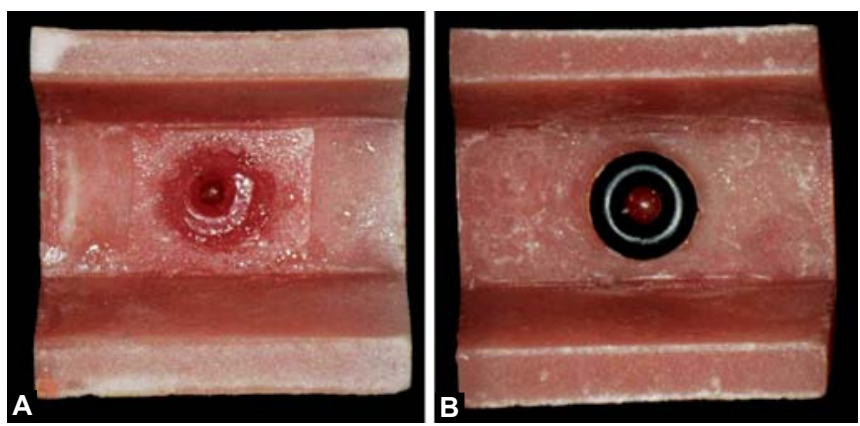

Figs $3 \mathbf{A}$ and $\mathbf{B}$ : Internal surface of the experimental base after combining: (A) soft liner and (B) O-ring

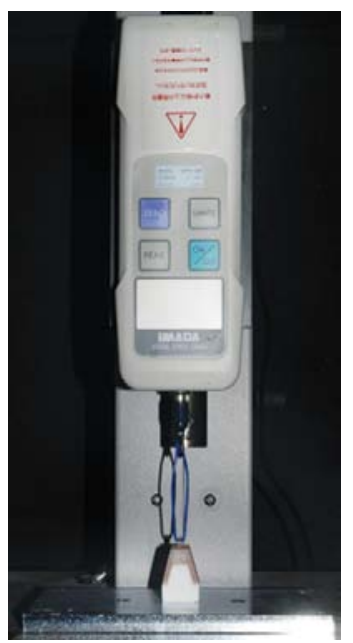

Fig. 4: Measuring device of retention force with digital force gauge

force. However, although the retention force increases with the diameter, because of the risk of exfoliation of the soft lining material, the increase in the size of the male connector should be as small as possible. The results show that the retention force of S-2.5 was higher than that of $\mathrm{R}-2.2$. Therefore, assuming that the retention force of $\mathrm{R}-2.2$ causes no problem in practical use, it is considered that the diameter of the male connector can be smaller than that of S-2.5. Minimizing the male connector size can relax the restrictions on denture design. It is necessary to investigate

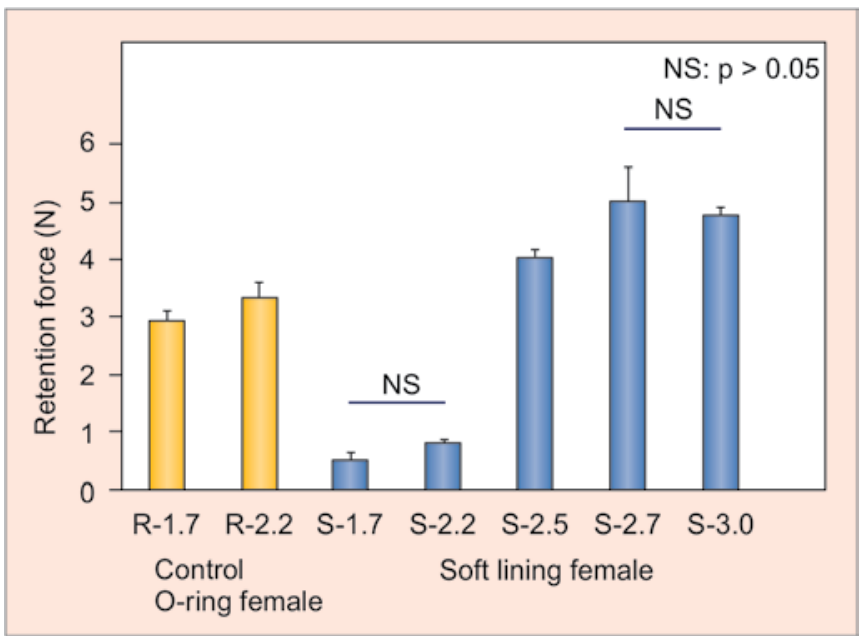

Fig. 5: Comparison of maximum retention forces

whether the durability of the soft lining material as the female connector poses no problem compared with other systems ${ }^{15}$ and to experimentally confirm the permissible angle of the parallelism of fixtures using models. It is considered that clarification of these points will facilitate clinical application of the attachment system with soft lining material.

If this system becomes practically established, then dentists will be able to readily produce IOD using soft lining materials. It will be unnecessary for them to pay attention to a strict angle restriction and junction between the male connector and female connector when multiple attachments are used. Furthermore, the soft lining material attachment is simple, inexpensive as compared to buying female attachemnt separately, and allows straightforward replacement. Damage due to exfoliation ${ }^{16}$ and degradation ${ }^{17}$ of soft lining materials in the attachment area after a long-term course ${ }^{18}$ can be quickly repaired during regular maintenance after implantation. We believe that this study confirms the high potential of the attachment system using soft lining materials for the overdentures. But we do not know something about its durability, disadvantages of other attachment systems and so on. Future studies are desired for practically use, for example measurement the retention with two or more attachments, long-term results and so on.

\section{CONCLUSION}

Under the experimental conditions considered in this study, when the diameter of the spherical male connector exceeded $2.5 \mathrm{~mm}$, a retention force equal to or higher than that of the rubber O-ring attachment system was achieved. It is, thus, suggested that the proposed attachment system with soft lining material for IOD can provide a clinically sufficient retention force when the diameter of the male connector exceeds $2.5 \mathrm{~mm}$. 


\section{REFERENCES}

1. Feine JS, Carlsson GE, Awad MA, Chehade A, Duncan WJ, Gizani S, Head T, Lund JP, MacEntee M, Mericske-Stern R, et al. The McGill consensus statement on overdentures. Mandibular two-implant overdentures as first choice standard of care for edentulous patients. Montreal, Quebec, May 24-25, 2002. Int J Oral Maxillofac Implants 2002 Jul-Aug;17(4):601-602.

2. Carlsson GE, Kronström M, de Baat C, Cune M, Davis D, Garefis P, Heo SJ, Jokstad A, Matsuura M, Närhi T, et al. A survey of the use of mandibular implant overdentures in 10 countries. Int J Prosthodont 2004 Mar-Apr;17(2):211-217.

3. Büttel AE, Lüthy H, Sendi P, Marinello CP. Wear of ceramic and titanium ball attachments in subjects with an implant-retained overdenture: a controlled clinical trial. J Prosthet Dent 2012 Feb;107(2):109-113.

4. Porter JA Jr, Petropoulos VC, Brunski JB. Comparison of load distribution for implant over denture attachments. Int J Oral Maxillofac Implants 2002 Sep-Oct;17(5):651-662.

5. Takeshita S, Kanazawa M, Minakuchi S. Stress analysis of mandibular two-implant overdenture with different attachment systems. Dent Mater J 2011 Nov;30(1):928-934.

6. Kawashima I, Nishizaki H, Okazaki J. Generation of retentive force by electroformed telescope crowns. J Osaka Dent Univ 2009;43:19-28.

7. Lee MW, Baum L, Pence B Sr, Hererra W. O-ring coping attachments for removable partial dentures. J Prosthet Dent 1995 Sep;74(3):235-241.

8. Guttal SS, Tavargeri AK, Nadiger RK, Thakur SL. Use of an implant O-ring attachment for the tooth supported mandibular overdenture: a clinical report. Eur J Dent 2011 Jul;5(3):331-336.

9. Winkler S, Piermatti J, Rothman A, Siamos G. An overview of the O-ring implant overdenture attachment: clinical reports. J Oral Implantol 2002;28(2):82-86.

10. Baker PS, Ivanhoe JR. Fabrication of occlusal device for protection of implant overdenture abutments with O-ring attachments. J Prosthet Dent 2003 Dec;90(6):605-607.

11. Kobayashi M, Ohkubo C, Suzuki Y, Aoki T, Sato J, Hosoi T. Retentive force of O-ring attachment to use Immediate Provisional Implant (IPI)-retained overdenture. Eur J Prosthodont Restor Dent 2005 Dec;13(4):147-149.

12. Elsyad MA, Gebreel AA, Fouad MM, Elshoukouki AH. The clinical and radiographic outcome of immediately loaded mini implants supporting a mandibular overdenture. A 3-year prospective study. J Oral Rehabil 2011 Nov;38(11):827-834.

13. Jofre J, Cendoya P, Munoz P. Effect of splinting mini-implants on marginal bone loss: a biomechanical model and clinical randomized study with mandibular overdentures. Int J Oral Maxillofac Implants 2010 Nov-Dec;25(6):1137-1144.

14. Koike T, Ishizaki K, Ogami K, Ueda T, Sakurai K. Influence of anterior palatal coverage on perception and retention in complete dentures. J Prosthet Dent 2011 Apr;105(4):272-279.
15. Naert I, Alsaadi G, Quirynen M. Prosthetic aspects and patient satisfaction with two-implant-retained mandibular overdentures: a 10-year randomized clinical study. Int J Prosthodont $2004 \mathrm{Jul}-$ Aug;17(4):401-410.

16. Madan N, Datta K. Evaluation of tensile bond strength of heat cure and autopolymerizing silicone-based resilient denture liners before and after thermocycling. Indian J Dent Res 2012 JanFeb;23(1):64-68.

17. Kimoto S, Kimoto K, Murakami H, Gunji A, Ito N, Kawai Y. Survival analysis of mandibular complete dentures with acrylicbased resilient liners. Gerodontology 2013 sep;30(3):187-193.

18. Cakarer S, Can T, Yaltirik M, Keskin C. Complications associated with the ball, bar and Locator attachments for implant-supported overdentures. Med Oral Patol Oral Cir Bucal 2011 Nov 1;16(7):e953-e959.

\section{ABOUT THE AUTHORS}

\section{Takashi Koike}

Assistant Professor, Department of Removable Prosthodontics and Gerodontology, Tokyo Dental College, 1-2-2 Masago, Mihama-ku Chiba, Japan

Correspondence Address: 1-2-2 Masago, Mihama-ku, Chiba 2618502, Japan, Phone: +81-43-270-3933, Fax: +81-43-270-3935, e-mail: koiketakashi@tdc.ac.jp

\section{Takayuki Ueda}

Associate Professor, Department of Removable Prosthodontics and Gerodontology, Tokyo Dental College, 1-2-2 Masago, Mihama-ku Chiba, Japan

\section{Syuutarou Noda}

Resident, Department of Removable Prosthodontics and Gerodontology Tokyo Dental College, 1-2-2 Masago, Mihama-ku, Chiba, Japan

\section{Koichiro Ogami}

Senior Assistant Professor, Department of Removable Prosthodontics and Gerodontology, Tokyo Dental College, 1-2-2 Masago, Mihama-ku Chiba, Japan

\section{Pravinkumar G Patil}

Assistant Professor, Department of Prosthodontics, Government Dental College and Hospital, Nagpur, Maharashtra, India

\section{Kaoru Sakurai}

Professor and Chair, Department of Removable Prosthodontics and Gerodontology, Tokyo Dental College, 1-2-2 Masago, Mihama-ku Chiba, Japan 